\title{
The machanism of neuroinflammation in Parkinson's disease: the involvement of integrin
}

\author{
Dan Zhang ${ }^{1}$, Liyan $\mathrm{Hou}^{2}$, Qingshan Wang ${ }^{2}$, Xiuqi Bao' \\ ${ }^{1}$ Institute of Materia Medica, Chinese Academy of Medical Scienses \& Peking Union Medical College, China, ${ }^{2}$ Dalian \\ Medical University, China
}

Background:The activation of microglial NADPH oxidase (NOX2) induced by alpha synuclein has been implicated in Parkinson's disease (PD). However, how alpha synuclein activates NOX2 remains unclear. The obscure of mechanisms greatly hampers the development of novel therapeutic strategies. Therefore, elucidating the potential mechanisms is urgently needed.

Methods: In the present study, we sought to identify the molecular mechanisms involved in alpha Syn-induced NOX2 activation by using microglial cultures and transgenic mice. The potential roles of both TLR2 and integrin in alpha Syninduced activation of NOX2 were investigated both in vitro and in vivo. The Rho signaling pathway was subsequently studied to reveal the detailed mechanism.

Results: In this study, we found that blocking integrin CD11b but not TLR2 attenuated alpha synuclein-induced NOX2 activation in microglia. The involvement of CD11b in a synuclein-induced activation of NOX2 was further confirmed in CD11b knockout microglia by showing reduced membrane translocation of NOX2 cytosolic subunit p47phox and superoxide production. Mechanistically, alpha synuclein bound to CD11b and subsequently activated Rho signaling pathway. Alpha Synuclein induced activation of RhoA and downstream ROCK but not Rac1 in a CD11b dependent manner. Moreover, siRNA mediated knockdown of RhoA impeded NOX2 activation in response to alpha synuclein. Furthermore, we found that inhibition of NOX2 failed to interfere with the activation of RhoA signaling and interactions between alpha synuclein and CD11b, further confirming that NOX2 was the downstream target of CD11b. Finally, we found that genetic deletion of CD11b abrogated alpha synuclein-induced NOX2 activatoin in vivo.

Conclusions: Taken together, our results indicated that integrin CD11b mediates alpha synuclein-induced NOX2 activation through a RhoA dependent pathway, providing not only a novel mechanistic insight but also a new potential therapeutic target for Parkinson's disease. 